\title{
Correspondence
}

\section{A Subspace-Based Approach to Blind Channel Identification in Pulse Shaping OFDM/OQAM Systems}

Helmut Bölcskei, Pierre Duhamel, and Rima Hleiss

\begin{abstract}
We introduce a subspace-based algorithm for the blind identification of time-dispersive channels in pulse shaping OFDM systems based on offset quadrature amplitude modulation (OFDM/OQAM). Our approach exploits cyclostationarity induced by the use of overlapping pulse shaping filters and uses second-order statistics only. The proposed method is similar in spirit to modulation-induced cyclostationarity. Our algorithm assumes symbol-rate sampling in the receiver and does not require knowledge of the channel order. We provide simulation results demonstrating the performance of the new method.
\end{abstract}

Index Terms-Blind equalization, cyclostationarity, offset QAM, OFDM, pulse shaping.

\section{INTRODUCTION}

Orthogonal frequency division multiplexing (OFDM) [3]-[6] is part of several telecommunications standards, such as satellite and terrestrial digital audio broadcasting (DAB), digital terrestrial TV broadcasting (DVB), asymmetric digital subscriber line (ADSL) for high-bit-rate digital subscriber services on twisted pair channels, and broadband indoor wireless systems.

Pulse Shaping OFDM Systems: Recently, it has been pointed out in [5]-[7] that OFDM systems based on offset quadrature amplitude modulation (OFDM/OQAM) [8] bypass a major disadvantage of OFDM schemes based on ordinary QAM, namely, the fact that time-frequency well-localized (and hence dispersion-robust) pulse-shaping filters are prohibited in the case of critical time-frequency density, where spectral efficiency is maximal. Pulse-shaping OFDM/OQAM systems are therefore well suited for wireless high-data-rate applications. Besides dispersion robustness, further advantages of pulse shaping are reduced out-of-band emission in wireless OFDM systems and reduced sensitivity to synchronization errors. However, since OFDM/OQAM systems do not employ temporal guard regions such as a cyclic prefix $(\mathrm{CP})$ [3], equalization has to be performed.

Channel Identification: Usually, channel identification in OFDM systems is accomplished using training data, which, however, results in a reduction of spectral efficiency. This reduction, including the overhead due to the $\mathrm{CP}$, can be up to $50 \%$. In this paper, we show that OFDM/OQAM signals contain sufficient structure to accomplish blind channel identification using second-order statistics only. Let us first briefly review previous work on that subject. In [9], a cyclostationarity-based algorithm for the blind identification of time-dispersive channels in CP OFDM systems has been introduced. Blind and semi-blind methods for channel identification in CP OFDM systems

Manuscript received June 2, 1999; revised December 8, 2000. The associate editor coordinating the review of this paper and approving it for publication was Prof. Michail K. Tsatsanis.

H. Bölcskei is with the Coordinated Science laboratory, University of Illinois at Urbana-Champaign, Urbana, IL 61801 USA (e-mail: bolcskei@comm.csl.uiuc.edu).

P. Duhamel and R. Hleiss are with CNRS/LSS, Supelec, Gif sur Yvette, France (e-mail: pierre.duhamel@1ss.supelec.fr).

Publisher Item Identifier S 1053-587X(01)03343-8. have been presented in [10]. A subspace-based algorithm for blind channel identification in systems employing more general filterbank precoders based on "short filters" (i.e., block transforms) has been proposed by Scaglione et al.in [11]. The algorithms in [9]-[11] exploit redundancy introduced by either the $\mathrm{CP}$ or a more general redundant precoder.

Contributions: In this correspondence, based on the results reported in [2] and [12], we develop a novel method for blind channel identification in pulse-shaping OFDM/OQAM systems. Our algorithm accomplishes blind channel identification from second-order statistics, even though no guard interval is introduced in the OFDM/OQAM transmitter, and no oversampling is performed in the receiver. Furthermore, the new method does not require knowledge of the channel order and does not impose restrictions on channel zeros.

Organization of the Paper: The rest of the paper is organized as follows. Section II briefly reviews pulse-shaping OFDM/OQAM systems and provides our assumptions and the problem statement. Section III shows that under quite general conditions, pulse shaping OFDM/OQAM signals are cyclostationary (CS), introduces the novel channel identification method, and discusses its properties. Finally, in Section IV, we present simulation results, and Section V concludes the paper.

\section{PULSE-SHAPING OFDM/OQAM SYSTEMS}

It has been shown in [13] that an OFDM/OQAM system with critical time-frequency density achieves the same pulse-shaping filter quality (in terms of time-frequency localization) as an ${ }^{1}$ OFDM/QAM system with $50 \%$ reduction in spectral efficiency. However, since OFDM/OQAM systems do not employ temporal guard regions, more complicated equalization than in CP OFDM systems is needed. A detailed discussion on the equalization of pulse-shaping OFDM/OQAM systems and a comparison of different equalizer structures appears in [14].

OFDM/OQAM Modulation: In an $M$-channel OFDM/OQAM system ( $M$ is assumed to be even), the transmit signal is given by

$$
\begin{aligned}
& x[n]= \sum_{k=0}^{M-1} x_{k}[n] \\
&=\sum_{k=0}^{M-1}\left[\sum_{l=-\infty}^{\infty} c_{k l}^{\mathcal{R}} g[n-l M] e^{j(2 \pi / M) k(n-\alpha / 2)}\right. \\
&+\sum_{l=-\infty}^{\infty} j c_{k, l}^{\mathcal{I}} g[n+M / 2-l M] \\
&\left.\cdot e^{j(2 \pi / M) k(n-\alpha / 2)}\right]
\end{aligned}
$$

where $c_{k, l}^{\mathcal{R}}=\operatorname{Re}\left\{c_{k, l}\right\}$, and $c_{k, l}^{\mathcal{I}}=\operatorname{Im}\left\{c_{k, l}\right\}$ are the real and imaginary parts of the data symbols $c_{k, l}$, respectively, $g[n]$ is the transmitter pulse shaping filter of length $L_{g}$, and $\alpha \in[0, M-1]$. An OFDM/OQAM transceiver is depicted in Fig. 1. The real-valued filter

\footnotetext{
${ }^{1}$ Note that the symbol constellation is not really limited to QAM. We chose
} the terminology of OFDM/QAM to emphasize the difference to OFDM/OQAM. 
$g[n]$ is orthogonal if it satisfies $g[\alpha+(2 r+1) M / 2-n]=g[n]$ for some $r \in \mathbb{Z}$, and

$$
A^{(g, g)}\left[l M, 2 \frac{k}{M}\right)=\delta[l] \delta[k], \quad l \in \mathbb{Z}, k \in[0, M / 2-1]
$$

with the auto-ambiguity function $A^{(g, g)}[k, \theta)=\sum_{n=-\infty}^{\infty} g[n] g[n-$ $k] e^{-j 2 \pi n \theta}$.

Assumptions and Problem Statement: Throughout the paper, we assume perfect synchronization, i.e., the received OFDM signal is given by

$$
r[n]=\sum_{s=0}^{L_{d}-1} x[n-s] d[s]+\rho[n]
$$

where $d[n]$ denotes the (complex-valued) time-dispersive channel of length $L_{d}$, and $\rho[n]$ is a wide-sense-stationary noise process, independent of the data symbols $c_{k, l}$. The correlation function of the noise process is given by ${ }^{2} c_{\rho}[\tau]=\mathcal{E}\left\{\rho[n] \rho^{*}[n-\tau]\right\}$. The symbols $c_{k, l}$ are drawn from a finite-alphabet complex constellation and satisfy $\mathcal{E}\left\{c_{k, l}^{\mathcal{R}} c_{k^{\prime}, l^{\prime}}^{\mathcal{R}}\right\}=\sigma_{c, R}^{2} \delta\left[k-k^{\prime}\right] \delta\left[l-l^{\prime}\right]$, $\mathcal{E}\left\{c_{k, l}^{\mathcal{I}} c_{k^{\prime}, l^{\prime}}^{\mathcal{I}}\right\}=\sigma_{c, I}^{2} \delta\left[k-k^{\prime}\right] \delta\left[l-l^{\prime}\right]$, and $\mathcal{E}\left\{c_{k, l}^{\mathcal{R}} c_{k^{\prime}, l^{\prime}}^{\mathcal{I}}\right\}=0$. We furthermore assume that the receiver knows the pulse shaping filter $g[n]$ and $\sigma_{c, R}^{2}$ and $\sigma_{c, I}^{2}$, respectively. This knowledge constitutes the basis for the blind identification algorithm discussed in Section III. We want to identify the channel impulse response $d[n]$ from second-order statistics of the received signal $r[n]$, aiming at estimators that do not require knowledge of the distribution of $\rho[n]$, and apply to the blind (nondata-aided) scenario.

\section{CYClOSTATIONARITY AND BLIND IDENTIFICATION ALGORITHM}

While it is conceivable that the use of temporal guard regions such as the $\mathrm{CP}$ (and hence redundancy) makes blind channel identification possible [9], this is less obvious in pulse shaping OFDM/OQAM systems, where no guard regions are used. We will see that if no pulse shaping is employed in OFDM/OQAM systems (i.e., the pulse shaping filter is a rectangular function of length $M$ ), the transmit signal is stationary rather than CS, which implies that nonminimum-phase channels cannot be identified using second-order statistics only. Obviously, overlapping pulse shaping filters act as a form of precoder that introduces cyclostationarity. This idea is close in spirit to modulation-induced cyclostationarity [1], [2], where the transmitted data sequence is multiplied by a deterministic periodic sequence to render the symbol-rate sampled received signal CS. The price to be paid for modulation-induced cyclostationarity and for cyclostationarity induced by pulse shaping is that for a given average transmit power, the minimum distance between symbols decreases, and hence, in order to achieve a given bit error rate, a higher average transmit power is required. ${ }^{3}$ Therefore, even though the transmission rate is kept constant, there is a loss in spectral efficiency.

\section{A. Cyclostationarity in OFDM/OQAM Systems}

In the following, the correlation function of a (nonstationary) stochastic process $x[n]$ is defined as $c_{x}[n, \tau]=\mathcal{E}\left\{x[n] x^{*}[n-\tau]\right\}$, where $\tau$ is an integer lag parameter. ${ }^{4}$ The signal $x[n]$ is said to be second-order CS with period $M$ if $c_{x}[n, \tau]=c_{x}[n+M, \tau]$ [15], [16].

$2 \mathcal{E}$ denotes the expectation operator.

${ }^{3}$ The authors would like to thank Reviewer A for pointing out these conclusions on modulation-induced cyclostationarity and on cyclostationarity induced by pulse shaping.

${ }^{4}$ For stationary processes, the correlation function $c_{x}[n, \tau]$ is a function of the lag parameter $\tau$ only.

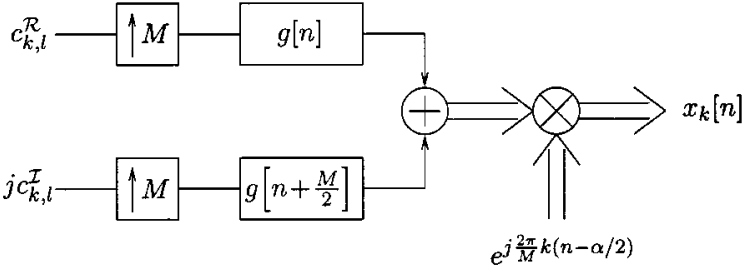

(a)

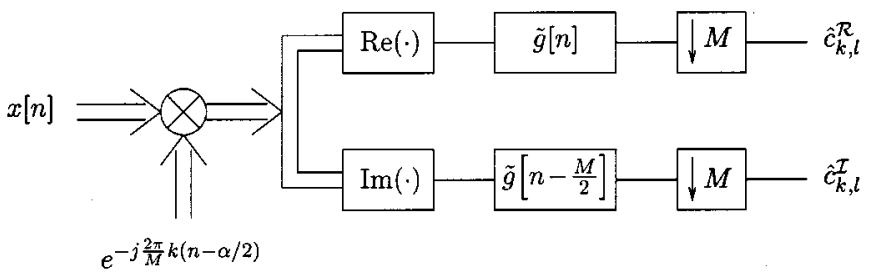

(b)

Fig. 1. Pulse-shaping OFDM/OQAM system $(\tilde{g}[n]=g[-n])$. (a) $k$ th transmitter subchannel. (b) $k$ th receiver subchannel.

Using (1), it is somewhat tedious but straightforward to show that the correlation function of $x[n]$ is given by

$$
c_{x}[n, \tau]=\delta_{M}[\tau]\left[\sigma_{c, R}^{2} a_{M}^{(\tau)}[n]+\sigma_{c, I}^{2} a_{M}^{(\tau)}\left[n+\frac{M}{2}\right]\right]
$$

where $\delta_{M}[\tau]=M \sum_{s=-\infty}^{\infty} \delta[\tau-s M]$, and $a_{M}^{(\tau)}[n]=$ $\sum_{l=-\infty}^{\infty} g[n-l M] g[n-l M-\tau]$. From $a_{M}^{(\tau)}[n+M]=a_{M}^{(\tau)}[n]$, it follows that $c_{x}[n+M, \tau]=c_{x}[n, \tau]$. If no pulse shaping is employed, i.e., $g[n]=1 / \sqrt{M}$ for $n \in[0, M-1]$ and 0 else, we obtain

$$
c_{x}[n, \tau]=\left(\sigma_{c, R}^{2}+\sigma_{c, I}^{2}\right) \delta[\tau]
$$

which implies that $x[n]$ is stationary. We conclude this subsection by noting that it is remarkable that pulse-shaping OFDM/OQAM signals are CS, although no guard interval is used.

\section{B. Blind Identification Algorithm}

We will next present the identification algorithm. Since the received signal $r[n]$ consists of a linear time-invariant filtered version of the transmit signal $x[n]$ and additive stationary noise $\rho[n]$, it will be CS whenever $x[n]$ is CS. It can now easily be shown that the Fourier series coefficients $C_{r}[k, \tau]=(1 / M) \sum_{n=0}^{M-1} c_{r}[n, \tau] e^{-j(2 \pi / M) k n}$ of $c_{r}[n, \tau]$ with respect to $n$ are given by

$$
\begin{aligned}
\mathcal{C}_{r}[k, \tau]= & \sum_{s=0}^{L_{d}-1} d[s] e^{-j(2 \pi / M) k s} \\
& \cdot \sum_{r=-\infty}^{\infty} d^{*}[s-\tau+r] \mathcal{C}_{x}[k, r]+c_{\rho}[\tau] \delta[k] \\
& k=0,1, \ldots, M-1
\end{aligned}
$$

with the Fourier series coefficients

$$
\mathcal{C}_{x}[k, \tau]=\frac{1}{M} \delta_{M}[\tau] A^{(g, g)}\left[\tau, \frac{k}{M}\right)\left[\sigma_{c, R}^{2}+(-1)^{k} \sigma_{c, I}^{2}\right]
$$

of $c_{x}[n, \tau]$. We will next need the cyclic spectrum of $r[n]$ defined by [15], [16] $\mathcal{S}_{r}[k, z)=\sum_{\tau=-\infty}^{\infty} \mathcal{C}_{r}[k, \tau] z^{-\tau}$. Using (5), we get ${ }^{5}$

$$
\mathcal{S}_{r}[k, z)=D\left(z e^{j(2 \pi / M) k}\right) \mathcal{S}_{x}[k, z) \tilde{D}(z)+S_{\rho}(z) \delta[k]
$$

${ }^{5}$ Here, $\tilde{D}(z)=D^{*}\left(1 / z^{*}\right)$ is the paraconjugate of $D(z)$. 
where $S_{x}[k, z)=\sum_{\tau-\infty}^{\infty} \mathcal{C}_{x}[k, \tau] z^{-\tau}$ denotes the cyclic spectrum of $x[n]$, and $S_{\rho}(z)=\sum_{\tau=-\infty}^{\infty} c_{\rho}[\tau] z^{-\tau}$. Now, following a procedure first suggested by Tong et al. in [12] and later used in [2] and [9], we can take two different cycles $k_{1} \in[1, M-1]$ and $k_{2} \in[1, M-1]$ to find

$$
\begin{aligned}
& D\left(z e^{j(2 \pi / M) k_{2}}\right) \mathcal{S}_{r}\left[k_{1}, z\right) \mathcal{S}_{x}\left[k_{2}, z\right) \\
& \quad-D\left(z e^{j(2 \pi / M) k_{1}}\right) \mathcal{S}_{r}\left[k_{2}, z\right) \mathcal{S}_{x}\left[k_{1}, z\right)=0 .
\end{aligned}
$$

Rewriting (8) in the time domain yields

$$
\begin{aligned}
\sum_{l=0}^{L_{d}-1} & {\left[a_{r, x}^{\left(k_{1}, k_{2}\right)}[n-l] e^{-j(2 \pi / M) k_{2} l}\right.} \\
& \left.\quad-a_{r, x}^{\left(k_{2}, k_{1}\right)}[n-l] e^{-j(2 \pi / M) k_{1} l}\right] d[l]=0, \quad n \in \mathbb{Z}
\end{aligned}
$$

with

$$
a_{r, x}^{(k, l)}[n]=\sum_{s=-\infty}^{\infty} \mathcal{C}_{r}[k, s] \mathcal{C}_{x}[l, n-s] .
$$

Using (6), it follows that $\mathcal{C}_{x}[k, \tau]=0$ for $|\tau|>L_{g}$. Furthermore, (5) implies that $\mathcal{C}_{r}[k, \tau]=0$ for $k \in[1, M-1]$ and $|\tau| \geq L_{g}+L_{d}-1$. We therefore have $a_{r, x}^{(k, l)}[n]=0$ for $|n| \geq 2 L_{g}+L_{d}-2$. From (7), it follows that the influence of stationary additive noise $\rho[n]$ (with arbitrary correlation function) will be reduced by considering nonzero cycles $k \in[1, M-1]$ only. In order to solve (9) for the channel $d[n]$, we rewrite it in vector-matrix form as

$$
\underbrace{\left[\mathbf{T}_{r, x}^{\left(k_{1}, k_{2}\right)} \mathbf{D}^{k_{2}}-\mathbf{T}_{r, x}^{\left(k_{2}, k_{1}\right)} \mathbf{D}^{k_{1}}\right]}_{\mathbf{S}_{r, x}^{\left(k_{1}, k_{2}\right)}} \mathbf{d}=\mathbf{0}
$$

with the $\left(4 L_{g}+3 L_{d}-6\right) \times L_{d}$ Toeplitz matrices $\mathbf{T}_{r, x}^{(k, l)}$ with first row $\left[a_{r}^{(k, l)}\left[-2 L_{g}-L_{d}+3\right] 0 \cdots 0\right]$ and first column $\left[a_{r, x}^{(k, l)}\left[-2 L_{g}-L_{d}+3\right] \cdots a_{r, x}^{(k, l)}\left[2 L_{g}+L_{d}-3\right] 0 \cdots 0\right]$, and the $L_{d} \times L_{d}$ diagonal matrix $\mathbf{D}=\operatorname{diag}\left\{e^{-j(2 \pi / M) l}\right\}_{l=0}^{L_{d}-1}$. Furthermore, $\mathbf{d}=\left[\begin{array}{llll}d[0] & d[1] & \cdots & d\left[L_{d}-1\right]\end{array}\right]^{T}$ and $\mathbf{0}$ denotes the $\left(4 L_{g}+3 L_{d}-6\right) \times 1$ all-zero vector. From (11), it follows that the channel $d[n]$ can be uniquely recovered up to a complex scalar if the matrix $\mathbf{S}_{r, x}^{\left(k_{1}, k_{2}\right)}$ has nullity one. Using [2, Th. 1], it can be shown that the channel is uniquely identifiable within a complex scalar (irrespective of channel zeros) if and only if there is no $l \in\left[1, L_{d}-1\right]$ such that $e^{-j(2 \pi / M)\left(k_{1}-k_{2}\right) l}=1$. If we choose $k_{1}$ and $k_{2}$ such that $\left|k_{1}-k_{2}\right|=K$, identifiability is guaranteed, irrespective of channel zeros for $L_{d} \leq M / K$. Since OFDM symbols are very long in practice (i.e., typically $L_{d} \leq M / 4$ ), this condition can easily be met.

If the true correlations $\mathcal{C}_{r}[k, \tau]$ are known, the channel can be found as the unique null eigenvector of ${ }^{6}$ the $L_{d} \times L_{d}$ matrix $\mathbf{S}_{r, x}^{\left(k_{1}, k_{2}\right)^{H}} \mathbf{S}_{r, x}^{\left(k_{1}, k_{2}\right)}$, and the channel order can be estimated from $\mathbf{S}_{r, x}^{\left(k_{1}, k_{2}\right)}$ as the minimum order $L_{d}$ for which the matrix $\mathbf{S}_{r, x}^{\left(k_{1}, k_{2}\right)}$ has nullity one. In practice, however, $\mathcal{C}_{r}[k, \tau]$ has to be estimated from a finite data record $\{r[n]\}_{n=0}^{L-1}$ of length $L$ according to [15]-[17]

$$
\hat{\mathcal{C}}_{r}[k, \tau]=\frac{1}{L} \sum_{n=0}^{L-1} r[n] r^{*}[n-\tau] e^{-j(2 \pi / M) k n} .
$$

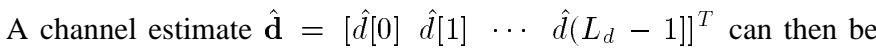
obtained by solving

$$
\hat{\mathbf{d}}=\arg \min _{\|\mathbf{d}\|=1}\left\|\hat{\mathbf{S}}_{r, x}^{\left(k_{1}, k_{2}\right)} \mathbf{d}\right\|^{2}
$$

${ }^{6}$ The superscript $H$ stands for conjugate transposition. where $\hat{\mathbf{S}}_{r, x}^{\left(k_{1}, k_{2}\right)}$ is an estimate of $\mathbf{S}_{r, x}^{\left(k_{1}, k_{2}\right)}$ obtained by replacing $\mathcal{C}_{r}[k, \tau]$ in $(10)$ by the estimates $\hat{\mathcal{C}}_{r}(k, \tau)$. Recall that since we assumed that $g[n], \sigma_{c, R}^{2}$ and $\sigma_{c, I}^{2}$ are known, the Fourier series coefficients $\mathcal{C}_{x}[k, \tau]$ are fully determined. In practice, however, the statistics $\hat{C}_{x}[k, \tau]$ contain an estimation error. Therefore, the solution of (13) is the eigenvector of $\hat{\mathbf{S}}_{r, x}^{\left(k_{1}, k_{2}\right)^{H}} \hat{\mathbf{S}}_{r, x}^{\left(k_{1}, k_{2}\right)}$ associated with the smallest eigenvalue.

Aspects of the Algorithm: The performance of the algorithm will critically depend on the choice of the cycles $k_{1}$ and $k_{2}$. An important aspect to be considered in the choice of the cycles is motivated by the following observation. According to (6), the Fourier series coefficients $\mathcal{C}_{x}[k, \tau]$ are directly proportional to the auto-ambiguity function $A^{(g, g)}[\tau, k / M)$. Now, since, for $g[n]$, a lowpass function $A^{(g, g)}[\tau, k / M)$ as a function of $k$ is a lowpass function as well, the magnitude of $\mathcal{C}_{x}[k, \tau]$ will decrease for increasing cycle index $k$. For small data record lengths, all cycles will contain an estimation error due to stationary noise. However, since $\left|\mathcal{C}_{x}[k, \tau]\right|$ and, hence, $\left|\mathcal{C}_{r}[k, \tau]\right|$ decrease as a function of $k$, the higher order cycles will be more prone to estimation errors than the lower order cycles. Therefore, if the pulse shaping filter's bandwidth is small, only the first few cycles should be used in the estimation.

We also observed that increasing the pulse-shaping filter length degrades the estimator performance. This can be explained as follows. The maximum lag in $\mathcal{C}_{r}[k, \tau]$ used for estimating the channel is $\tau_{\max }=L_{g}+L_{d}-2$, i.e., it increases for increasing pulse-shaping filter length $L_{g}$. Now, it has been observed previously in [17] that the accuracy of the estimated cyclic correlations $\hat{\mathcal{C}}_{r}[k, \tau]$ decreases for lags $\tau$ far away from the origin. This is due to reduced averaging [see (12)]. Therefore, the estimate $\hat{\mathbf{S}}_{r, x}^{\left(k_{1}, k_{2}\right)}$ will be more inaccurate for long pulse-shaping filters. In summary, longer pulse-shaping filters require more averaging (i.e., longer data record lengths). This observation, together with the lack of a guard region in OFDM/OQAM systems, is the reason for the slightly worse performance of our algorithm when compared with the results reported for the CP OFDM case in [9]. Indeed, it was demonstrated in [9] that increasing the length of the $\mathrm{CP}$ improves the estimator performance.

We finally note that since the proposed algorithm is a statistical approach, it generally requires very long data records to obtain good estimates. An asymptotic performance analysis of our OFDM/OQAM channel estimator is an interesting topic for future research. We note that for periodic modulation precoders, such an analysis appears in [18].

\section{Simulation Results}

We simulated an OFDM/OQAM system with $M=8$ channels and pulse-shaping filter length 16 (i.e., overlapping factor 2). Fig. 2 shows the pulse-shaping filter, which has been designed using techniques reported in [7]. In both simulation examples, we used the cycles $k_{1}=$ 1 and $k_{2}=M-k_{1}=7$ to estimate the five-tap channel $\mathbf{d}=$

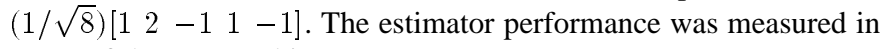
terms of the average bias

$$
\frac{1}{I L_{d}} \sum_{l=0}^{L_{d}-1}\left|\sum_{i=0}^{I-1}\left[\hat{d}^{(i)}[l]-d[l]\right]\right|
$$

and the mean square error (MSE)

$$
\frac{1}{I L_{d}\|d\|^{2}} \sum_{i=0}^{I-1}\left\|\hat{\mathbf{d}}^{(i)}-\mathbf{d}\right\|^{2}
$$

where $I$ denotes the number of Monte Carlo trials. The data symbols were i.i.d. 64-QAM symbols. The signal-to-noise-ratio (SNR) was de- 

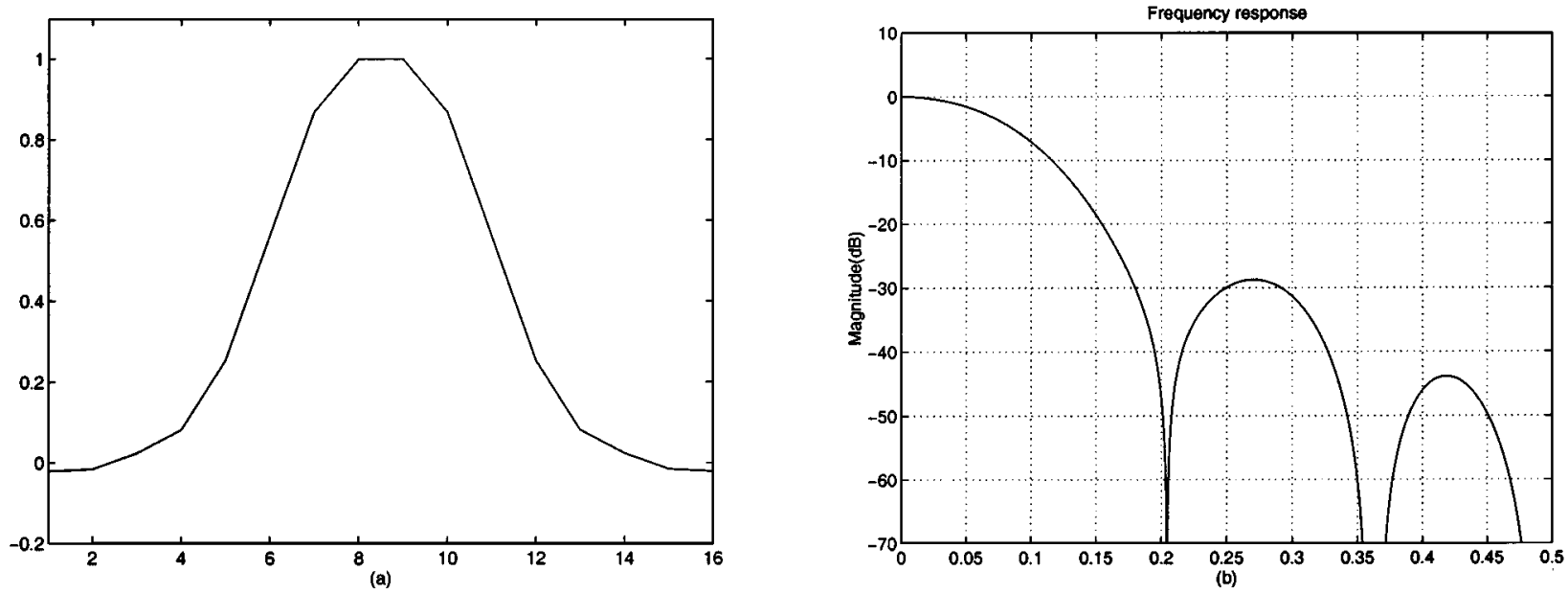

Fig. 2. OFDM/OQAM pulse shaping filter of length 16. (a) Impulse response. (b) Transfer function.
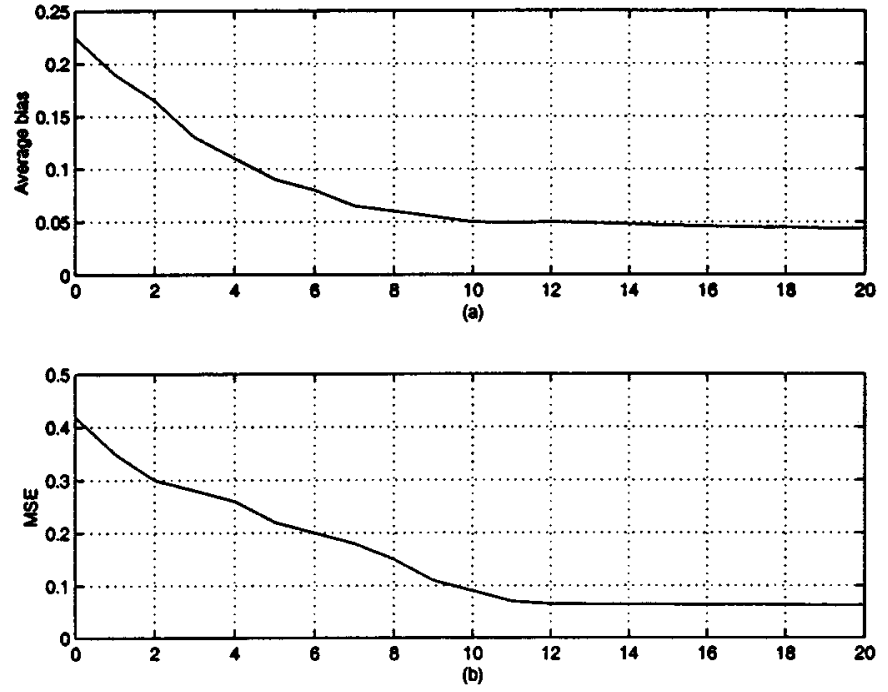

Fig. 3. Blind channel identification in an OFDM/OQAM system with $M=8$. (a) Average bias. (b) MSE of the channel estimator as a function of SNR in decibles.

fined as

$$
\mathrm{SNR}=10 \log \left(\frac{\sigma_{c, R}^{2}+\sigma_{c, I}^{2}}{\sigma_{\rho}^{2}}\right)
$$

where $\sigma_{\rho}^{2}$ is the variance of the white noise process $\rho[n]$. Furthermore, the symbol constellation was chosen such that ${ }^{7} \sigma_{c, R}^{2} / \sigma_{c, I}^{2}=4$. All results were obtained by averaging over $I=1000$ independent Monte Carlo trials, where each realization consisted of 1024 data symbols (i.e., 128 OFDM symbols).

Simulation Example 1: In the first simulation example, we computed the average bias and the MSE of our channel estimator [see Fig. 3(a) and (b)] as a function of SNR in decibels. The entire data record has been used to estimate the correlation function of the received signal. We can observe that the performance of the estimator improves with increasing SNR. Since we are not considering the cycle $k=0$, we would expect the performance of the estimator to be independent of the SNR. However, due to nonideal estimates of the CS statistics,

${ }^{7}$ If $\sigma_{c, R}^{2}=\sigma_{c, I}^{2}$, it follows from (6) that $\mathcal{C}_{x}[k, \tau]=0$ for $k$ odd. Since we are using the cycles $k_{1}=1$ and $k_{2}=7$ to estimate the channel, we have to make sure that this does not occur.
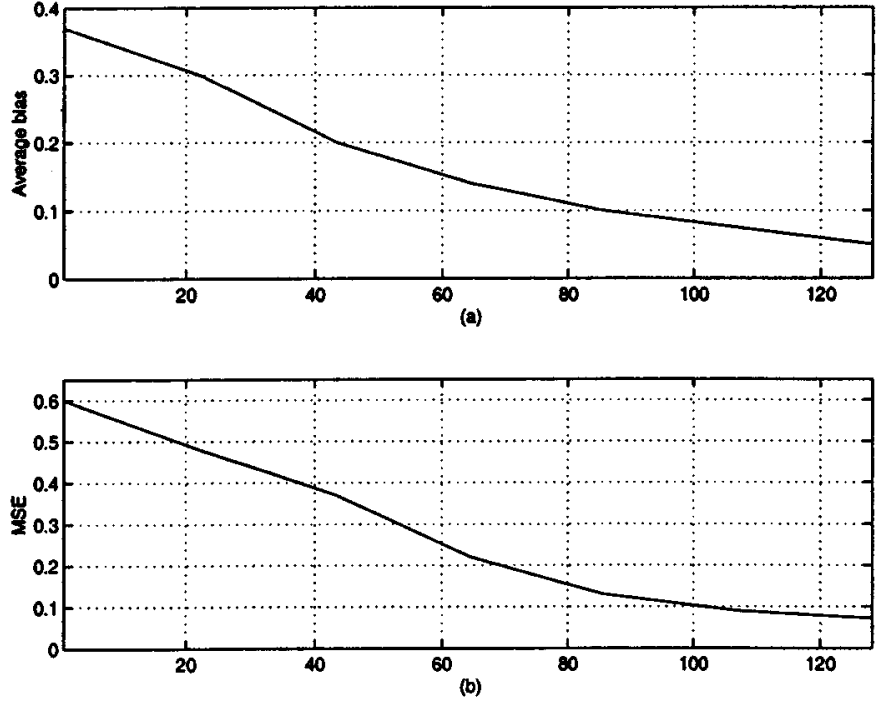

Fig. 4. Blind channel identification in an OFDM/OQAM system with $M=8$. (a) Average bias. (b) MSE of the channel estimator as a function of the data record length (specified in OFDM symbols).

this is obviously not the case. We can see that the estimator exhibits an error floor for large SNR. This error floor has also been observed in blind channel identification in CP OFDM systems [9] and in systems employing periodic modulation precoders [18].

Simulation Example 2: In the second simulation example, we investigate the effect of the length $L$ of the data record used to estimate the cyclic statistics $C_{r}[k, \tau]$ on the performance of the channel estimator. For $\mathrm{SNR}=15 \mathrm{~dB}$, Fig. 4(a) and (b) show the average bias and the MSE, respectively, of the channel estimator as a function of $L$. (Note that in Fig. 4, the length of the data record has been specified in OFDM symbols.) We can observe that the performance of the estimator improves with increasing data record length. However, it has to be noted that rather long data records are required to obtain good channel estimates.

\section{CONCLUSION}

We introduced an algorithm for blind second-order cyclostationary statistics-based channel identification in pulse-shaping OFDM/OQAM systems. We found that in the OFDM/OQAM case, despite the fact that no temporal guard region is used and no oversampling is performed in the receiver, blind channel identification from second-order statistics 
is possible. We emphasize, however, that the price to be paid for cyclostationarity induced by pulse shaping is a loss in spectral efficiency. Our approach assumes that the receiver knows the pulse-shaping filter; it does not require knowledge of the channel order and does not impose restrictions on channel zeros. A drawback of the algorithm is that it requires much averaging to arrive at good estimates. Finally, we presented simulation examples demonstrating the performance of the new algorithm.

\section{REFERENCES}

[1] A. Chevreuil and P. Loubaton, "Blind second-order identification of FIR channels: Forced cyclostationarity and structured subspace method," IEEE Signal Processing Lett., vol. 4, pp. 204-206, July 1997.

[2] E. Serpedin and G. B. Giannakis, "Blind channel identification and equalization with modulation induced cyclostationarity," IEEE Trans. Signal Processing, vol. 46, pp. 1930-1944, July 1998.

[3] A. Peled and A. Ruiz, "Frequency domain data transmission using reduced computational complexity algorithms," in Proc. IEEE ICASSP, Denver, CO, 1980, pp. 964-967.

[4] L. J. Cimini, "Analysis and simulation of a digital mobile channel using orthogonal frequency division multiplexing," IEEE Trans. Commun., vol. COMM-33, pp. 665-675, July 1985.

[5] R. Haas, "Application des transmissions à porteuses multiples aux communications radio mobiles," Ph.D. dissertation, Ecole Nat. Supérieure Télécommun. Paris, Paris, France, Jan. 1996.

[6] B. LeFloch, M. Alard, and C. Berrou, "Coded orthogonal frequency division multiplex," Proc. IEEE, vol. 83, pp. 982-996, June 1995.

[7] H. Bölcskei, P. Duhamel, and R. Hleiss, "Design of pulse shaping OFDM/OQAM systems for high data-rate transmission over wireless channels," in Proc. IEEE Int. Conf. Commun., Vancouver, BC, Canada, June 1999, pp. 559-564.
[8] B. Hirosaki, "An orthogonally multiplexed QAM system using the discrete Fourier transform," IEEE Trans. Commun., vol. COMM-29, pp. 982-989, July 1981.

[9] R. W. Heath and G. B. Giannakis, "Exploiting input cyclostationarity for blind channel identification in OFDM systems," IEEE Trans. Signal Processing, vol. 47, pp. 848-856, Mar. 1999.

[10] B. Muquet and M. de Courville, "Blind and semi-blind channel identification methods using second order statistics for OFDM systems," in Proc. IEEE ICASSP, Phoenix, AZ, Mar. 1999, pp. 2745-2748.

[11] A. Scaglione, G. B. Giannakis, and S. Barbarossa, "Redundant filterbank precoders and equalizers-Part II: Blind channel estimation, synchronization, and direct equalization," IEEE Trans. Signal Processing, vol. 47, pp. 2007-2022, July 1999 .

[12] L. Tong, G. Xu, B. Hassibi, and T. Kailath, "Blind identification and equalization based on second order statistics: A frequency domain approach," IEEE Trans. Inform. Theory, vol. 41, pp. 329-334, Jan. 1995.

[13] H. Bölcskei, "Design of pulse shaping filters for wireless OFDM systems," in Proc. SPIE Wavelet Applicat. Signal Image Processing III, vol. 3813, Denver, CO, July 1999, pp. 625-636.

[14] R. Hleiss, "Conception et egalization de nouvelles structures de modulations multiporteuses," Ph.D. dissertation, Ecole Nat. Supérieure Télécommun. Paris, Paris, France, Nov. 1999.

[15] W. A. Gardner, Ed., Cyclostationarity in Communications and Signal Processing. Piscataway, NJ: IEEE, 1995.

[16] G. B. Giannakis, "Cyclostationary signal analysis," in Digital Signal Processing Handbook, V. K. Madisetti and D. Williams, Eds: CRC, 1998.

[17] F. Gini and G. B. Giannakis, "Frequency offset and symbol timing recovery in flat-fading channels: A cyclostationary approach," IEEE Trans. Commun., vol. 46, pp. 400-411, Mar. 1998.

[18] A. Chevreuil, E. Serpedin, P. Loubaton, and G. B. Giannakis, "Blind channel identification and equalization using periodic modulation precoders: Performance analysis," IEEE Trans. Signal Processing, vol. 48, pp. 1570-1586, June 2000 